\title{
Design and Control of an Indoor Coaxial Helicopter
}

\section{Conference Paper}

Author(s):

Bouabdallah, Samir; Siegwart, Roland; Caprari, Gilles

Publication date:

2006

Permanent link:

https://doi.org/10.3929/ethz-a-010079391

Rights / license:

In Copyright - Non-Commercial Use Permitted 


\section{Design and Control of an Indoor Coaxial Helicopter}

\author{
Samir Bouabdallah and Roland Siegwart \\ Autonomous Systems Lab \\ ETH Zurich, Switzerland \\ Email: samir.bouabdallah@ieee.org
}

\author{
Gilles Caprari \\ GCtronic \\ Mendrisio, Switzerland \\ Email: g.caprari@ieee.org
}

\begin{abstract}
This paper presents the design and preliminary control of an indoor coaxial helicopter "CoaX" based on a concept of hybrid active and passive control. The robot is capable of passive roll and pitch stabilization against small disturbances while actively stabilizing the yaw and the altitude. We achieve omnidirectional horizontal motion by shifting the center of gravity. The structure is made out of lightweight polyurethane and carbon fiber. Preliminary tests show that "CoaX" is able to hover for short moments and the lateral control is effective.
\end{abstract}

\section{INTRODUCTION}

The interest in unmanned aerial robotics is rapidly growing thanks to the latest technological achievements which open the way to new applications such like aerial surveillance, search and rescue and aerial relays. The potential capabilities of these systems and the challenges behind are attracting the scientific and the industrial communities. The paper [1] outlined the development of a miniature autonomous flight control system and the creation of a multi-vehicle platform for experimentation and validation of multi-agent control algorithms. The document [2] presents several results in centimeter-scale quadrotor design and analysis. Another interesting development is the flapping concept presented in [3]. One similar development to CoaX is the MICOR, a coaxial helicopter presented in [4]. Moreover, a recent result from [5] is a $13.6 \mathrm{~cm}$ (5.3in) microhelicopter able to hover 3 minutes. It is remotely operated via a Bluetooth link and can carry an onboard camera. The Ecole Polytechnique Fédérale de Lausanne is also participating with several projects to this scientific endeavor [6]. In most of these applications the vehicle size is of a crucial importance thus, Miniature Flying Robots (MFR) are required as they can fly in complex or cluttered environments. Safety is also a big issue as MFRs have to fly close to humans. Moreover, safe MFRs could be used in education. These systems need to have good survivability, low power consumption and high payload. Miniature helicopters represent promising candidates thanks to their hovering capability which is essential in aerial communication relays for instance. The design of optimal MFR is a challenging task due to the interdependency of all design variables. This fact makes the choice of each variable strongly conditioned by the choice of all the others. Our approach is mainly applying innovative control technics to redesigned vehicles with very optimized mechanics. The idea is to miniaturize the robot in every redesign step, in order to push at each iteration the frontier of the feasible. In this paper we present the design of an indoor coaxial helicopter called



Fig. 1. The CoaX helicopter. (pict. Alain Herzog)

"CoaX" (see Fig. 1) based on a systematic design method for MFRs and we show the results achieved with a preliminary control scheme. The paper is organized in five sections. The next one describes the design process, the third one presents the preliminary control. In the fourth one we discuss the results and we make a conclusion and a review of the future work in the fifth section.

\section{COAX DESIGN}

\section{A. Configuration Selection}

1) Single rotor: Single rotor configuration is present in conventional helicopters with a main and a tail rotors. It provides good maneuverability and efficient aerodynamics. However, the tail boom and the large propeller size affects compactness and survivability.

2) Double rotor: Several concepts have two main rotors. This is true for coaxial and tandem helicopters. Coaxial configuration is by far more adapted to miniaturization thanks to its compactness, simplicity of the structure and its relative stability. However, it suffers from aerodynamical interference between the two propellers.

3) Quad rotor: This old concept with four propellers was tested in full size vehicles in the beginning of the century. Recently, there has been an interest in designing fully autonomous miniature quadrotors. This is due to their mechanical simplicity and good stability. However, quadrotors are difficult to miniaturize because of the aerodynamical problem.

4) Configuration choice: There exist a lot of other concepts but we decided to adopt a coaxial configuration mainly for 
its compactness and stability. The basic idea was to develop an MFR passively stable in roll and pitch while preserving good maneuverability in 3D translations. This has numerous advantages as listed below.

- Avoiding the usual slow dynamics of fixed pitch rotors: The rejection of disturbances is instantaneous (up to a given amplitude).

- Providing a better survivability in case of failure: As long as the propellers rotate, the CoaX can fly.

- Reducing considerably power consumption: Much less need for acceleration of rotational masses (rotors).

- Reduced controllers complexity: Less loading of processors.

5) Passive Stability Concepts: Two main concepts are possible to achieve passive stability in our context. The first one is based on the classical flybar system governing one or both propellers. The system has proven its efficiency especially for small scale helicopters. Its major drawback is the mechanical complexity and weight addition. The second concept is based on flexible propellers which behave basically like a flybar. The interest is in the mechanical simplicity and the stabilization efficiency.

6) Lateral Motion Concepts: There are a lot of possibilities to move our helicopter horizontally. The swashplate is the classical approach. It is reliable but it requires several small mechanical parts which are not simple to manufacture and thus, it is not suited for MFRs. Some other concepts take benefit from the downwash produced by the propellers. One can use a couple of flaps below the main rotors to deflect the airflow. The concept suffers from control complexity due to the nonlinearities introduced. Another possibility is to generate a force with Magnus effect by rotating cylinders perpendicular to the downwash, this concept is quite efficient and makes the control easier. A radically different approach is moving the center of gravity (CoG) which tilts the helicopter and thus the propellers. It is simple, reliable and easy to control. However, it requires a bit more energy that the previous concepts. In fact, many other concepts exist, they are often swashplate redesigns.

\section{B. Design Procedure}

The interdependency of the numerous design parameters makes it challenging to built and optimize such a system. Moreover, the scaling laws are unfavorable and thus, the sensors and actuators performance, the amount of energy and the embedded computation power are very limited. The designer has to evaluate other design variables concerning all the subsystems themselves. Taking a decision concerning all these variables requires to follow an appropriate methodology. We developed a practical method to handle the design problematic of a small scale rotorcraft by combining the theoretical knowledge of the system and a minimum of optimization results analysis, please refer to [7] for more details. The method is basically application oriented, it uses an iterative algorithm to output the best combination of components for a given constraints. The application to $\mathrm{CoaX}$ was constrained by a maximum of $200 \mathrm{~g}$ in mass and $300 \mathrm{~mm}$ in diameter. The

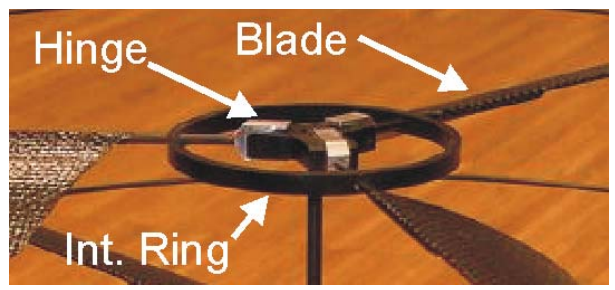

Fig. 2. Close view to the flexible propeller design. The internal ring avoids blades conning.

stability problem is one among many others. One approach to handle it is to make the system passively stable on the attitude but control actively the lateral motion. However, these two controls are often incompatible because of the strong coupling between rotations and translations. An appropriate control would handle the interaction between the two dynamical subsystems and make them cohabit.

\section{Passive Stability}

Passive stability was previously demonstrated in [8] and announced in [9]. Coax propellers provide a passive stability on the roll and pitch against small disturbances. This is achieved by allowing a relative flexibility between the rotor shaft and the propeller as shown in Fig. 2. So, any transient disturbance in the orientation or the horizontal translations will be countered by the propeller reaction. Every blade is free to rotate vertically but is constrained with an internal ring to avoid excessive conning.

\section{Augmented Stability}

As the passive stability is only for small disturbances, it was decided to augment it with active control when needed. In fact, shifting the CoG could also be activated to counter large disturbances in orientation. This is in fact coupled with lateral motion actuation as presented below. However, an appropriate control algorithm is able to handle this coupling and to distinguish the situation.

\section{E. Lateral Stability}

For the translations we considered in a first prototype an arrangement of 3 thrusters in $120^{\circ}$ in the horizontal plane. This makes it possible to achieve omnidirectional motion as depicted in Fig. 3 without a direct tilting of the main rotors. In the same time we used firstly three small propellers for the translations. This solution is simple but increases the system volume and power consumption while it decreases robustness and survivability. Secondly, we tested in the same arrangement three rotating cylinders taking benefit from the downwash to generate Magnus effect. This offers not only more compactness but also better efficiency and survivability than the small propellers. After several tests and brainstorming we converged to the conclusion that shifting the CoG by controlling the battery's position is the simplest solution for translations actuation on the second prototype as seen in Fig. 4. One may observe in Fig. 5 that we are using two servomotors (blue) and two semi-circular guides (grey) while the 

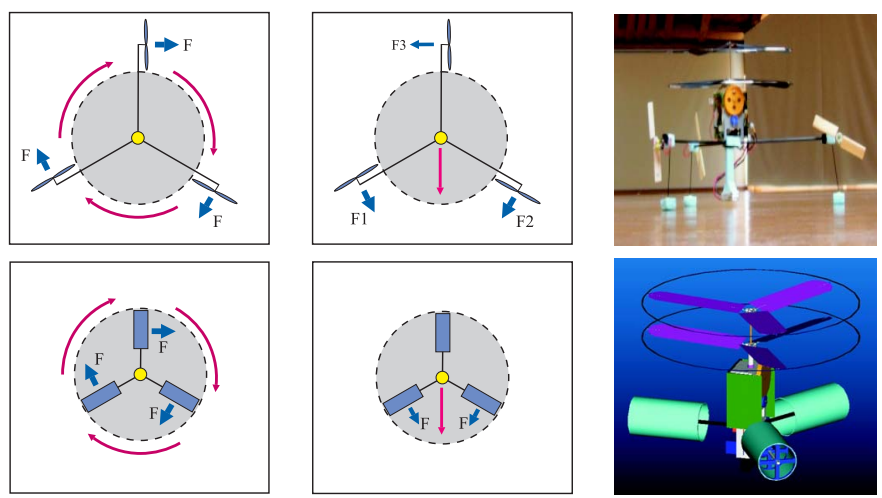

Fig. 3. First CoaX prototype with three small thrusters arranged at $120 \mathrm{deg}$ from each other and only one main motor. The upper design uses three small propellers and the lower three Magnus cylinders for horizontal motion.


Fig. 4. Shifting the CoG causes the helicopter to tilt. The resultant lateral force creates lateral motion.

battery is attached to the CoaX body through a kneecap mechanism (blue sphere). We can control each servo separately to move the battery in one axis, or simultaneously to achieve omnidirectional motion.

\section{F. Preliminary Testing}

The first experiments revealed some weakness in the main propellers (first prototype), in spite of which we were able to perform several passively stable short flights. In the second prototype we decided to use one motor for each main propeller for better yaw control. We selected a $12 \mathrm{~g}, 35 \mathrm{~W}$ brushless sensorless motor along with a 4:1 reduction gear and we developed a dedicated speed controller.

\section{G. The Configuration}

CoaX requires a hybrid passive/active control. Passive on the roll and pitch and active for the translations and the yaw. However, in case of strong disturbances, it is necessary to augment attitude control by CoG positioning. For this, we use

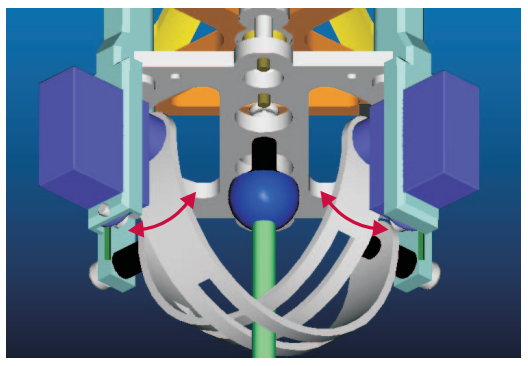

Fig. 5. CoaX CoG shifting system. The two servo-motors rotates according to the red arrows. a two axis inclinometer [10] for attitude determination. This sensor offers particularly a good signal to noise ratio along with a digital interface. On the other hand, we achieve yaw control by varying the rotors speed, while a standard MEMS gyroscope [11] is giving feedback. This was selected for its size, mass and good performance. Finally, we use a ranging sensor [12] to get the distance from the ground as an altitude sensor. This infrared device offers a narrower beam compared to an ultrasound one, which is suitable indoor. The structure is made out of lightweight polyurethane and carbon fiber rods. A $70 \mathrm{~g}, 900 \mathrm{mAh}$ Lithium-Polymer battery is used to power the system for about $20 \mathrm{~min}$.

\section{H. The Design Results}

The robot as a whole represents the result of the design methodology and fits the requirements. One can see CoaX mass and power distributions in Fig. 7. The total mass is about $200 \mathrm{~g}$ where the battery takes more than one-third and the actuators only one-fifth. All the actuators take obviously the lion's part, 25 of $27 \mathrm{~W}$ of the total power consumption. Obviously, the latter depends on flight conditions. Fig. 1 shows the real robot. The robot takes-off as soon as the
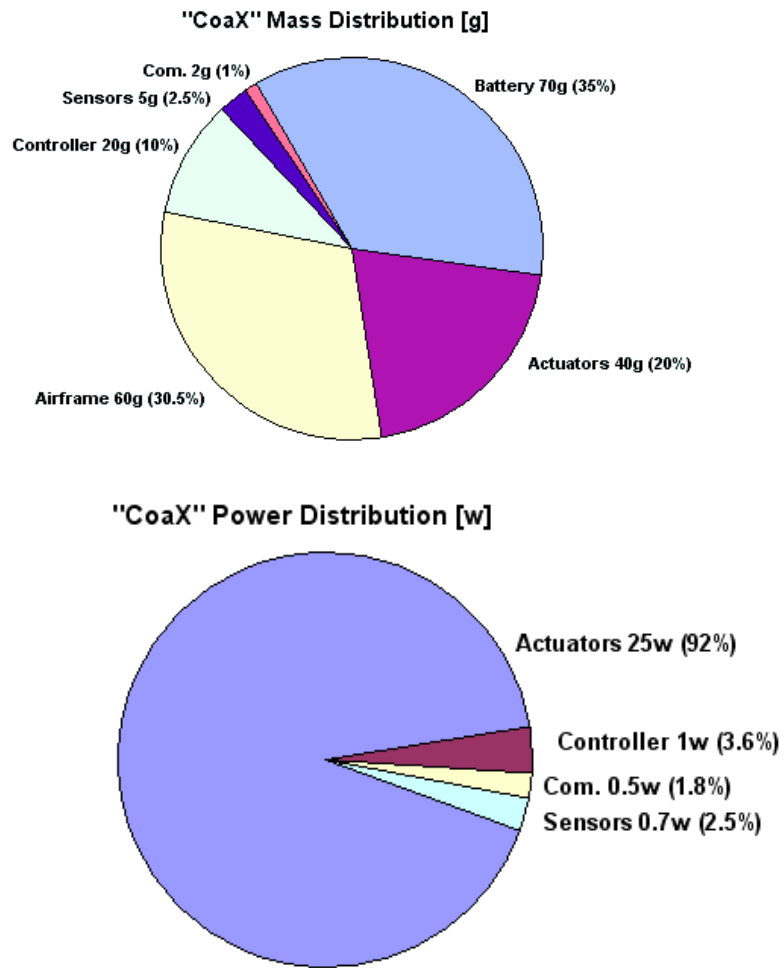

Fig. 7. Mass and power distributions in CoaX robot. The battery and the airframe mass take more than one-half the total mass.

propellers reaches about $145 \mathrm{rad} / \mathrm{s}$ as seen in Fig. 8. The passive stabilization is working fine but more investigation is still required. The power consumption in flight is about $25 \mathrm{~W}$ which is less than expected, the autonomy should be thus longer. 


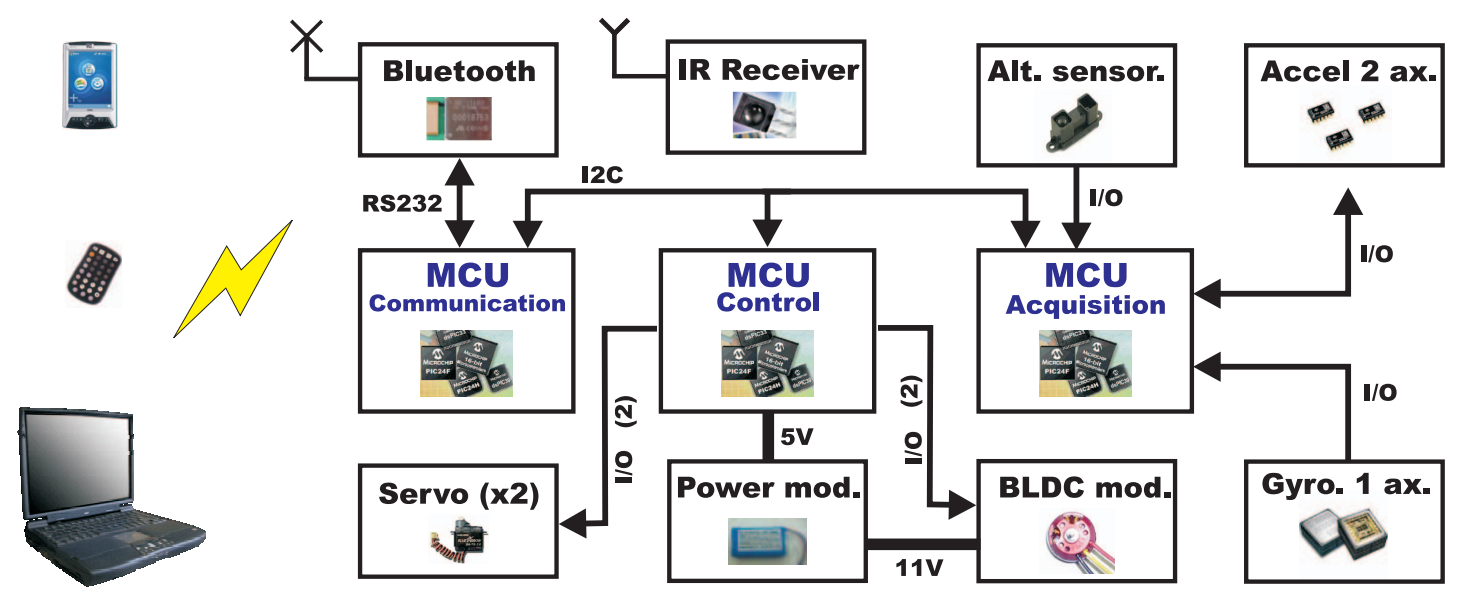

Fig. 6. CoaX Block Diagram. The processing is distributed over three micro-controllers linked with an $\mathrm{I}^{2} \mathrm{C}$ bus.



Fig. 9. Preliminary controller. All the sensor data are filtered. Simple PD controllers are applied for testing.

TABLE I

COAX FACTS

\begin{tabular}{r|c|c|c}
\hline parameter & symbol & value & unit \\
\hline Autonomy & $A u$ & 20 & $\mathrm{~min}$ \\
Inertial moment on $x$ & $I_{x x}$ & $0.976 \mathrm{e}-3$ & $\mathrm{~kg} \mathrm{~m}^{2}$ \\
Inertial moment on $y$ & $I_{y y}$ & $0.977 \mathrm{e}-3$ & $\mathrm{~kg} \mathrm{~m}^{2}$ \\
Inertial moment on $z$ & $I_{z z}$ & $0.233 \mathrm{e}-2$ & $\mathrm{~kg} \mathrm{~m}^{2}$ \\
Robot mass & $m$ & 0.2 & $\mathrm{~kg}$ \\
Robot span & $S p$ & 0.3 & $\mathrm{~m}$ \\
Robot height & $H$ & 0.25 & $\mathrm{~m}$ \\
\hline
\end{tabular}

\section{PReliminary CONTROL}

The control algorithm is running at $100 \mathrm{~Hz}$. The inputs are the sensor values and the reference points. The outputs are the speeds of the 2 motors and the positions of the 2 servos as depicted in Fig. 9. The 3 controllers (yaw, altitude, pitch and roll) can be enabled independently. If one of those is

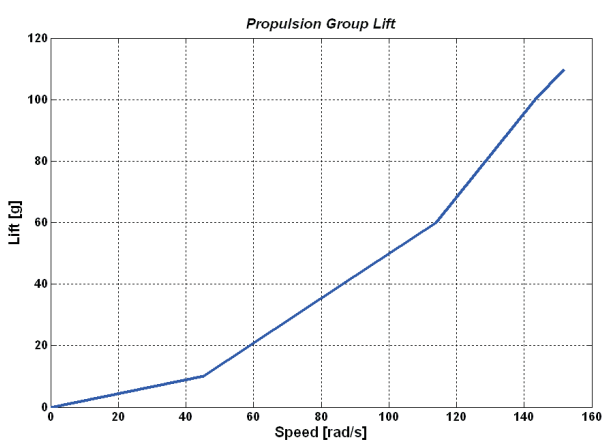

Fig. 8. Experiment: Single Propulsion group lift curve on CoaX.

not turned on, the relative values in the control formulas are simply set to zero. The sensor values are first filtered with a low-pass implemented with a moving average on the last 8 measures. The altitude sensor is pointing toward the floor with $45^{\circ}$ inclination, due to battery motion space. However, the 
measurement is compensated by software based on the attitude with a simplified formula. A speed and acceleration limitation is implemented on the motors allowing a single increment during a fixed time. As a result of all these computations the final motor values are executed. The user has access to the automatic control having the possibility to modify the reference points and to switch on/off one of the 3 independent loops.

\section{A. Active Control}

The yaw control uses the gyro as input sensor and acts on the differential speed between the 2 contra-rotating propellers. The sensor measurement is compared with the stable predefined value. The error enters a PI controller and the result is used to increase one propeller speed and decrease the other one. The altitude controller is driven by the IR distance sensor and acts on the same way to both propeller speed. First, the distance measured to the floor is corrected. Then, the computed altitude is compared with the wished one. The difference and a PD controller modify both motors speeds in order to keep a stable altitude. In automatic mode the effects of the altitude and yaw control are summed-up with the value established by the user and then passed to the motor speed controller. Roll and pitch are measured with the 2-axis inclinometer and can be influenced by moving the battery and consequently the $\mathrm{CoG}$ as seen in Fig. 10. The two axis are placed horizontally at $45^{\circ}$ with respect to the servos and thus the movement of one servo has influence on both roll and pitch angles. In automatic active control, the positions of the

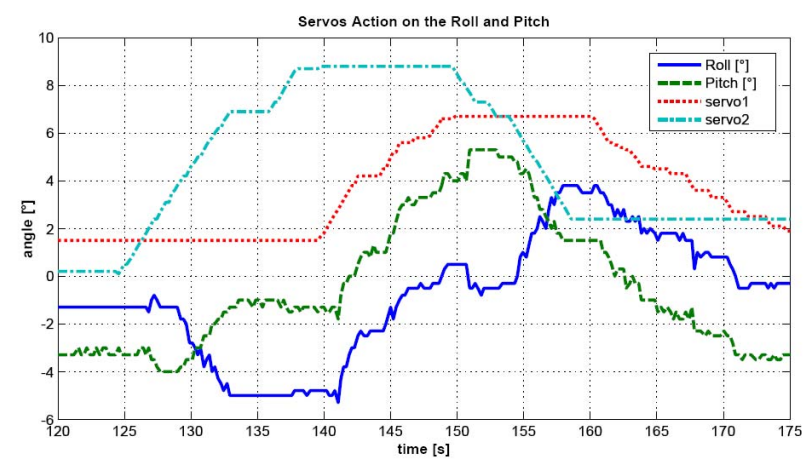

Fig. 10. Experiment: The effect of CoG shifting on the roll and pitch.

2 servos linked with the battery are the output of simple $\mathrm{P}$ controllers that help stabilizing the attitude. This controller tries to keep the inclination to a predefined stable position. In order to force CoaX to move horizontally, that stable position can be controlled by the user to achieve the desired horizontal motion. At the moment $\mathrm{CoaX}$ is not equipped with navigation sensors and thus, there is no possible automatic control in that sense. However, the configuration of the propellers limits the dynamics in the horizontal plane, this was observed during the several flying tests.

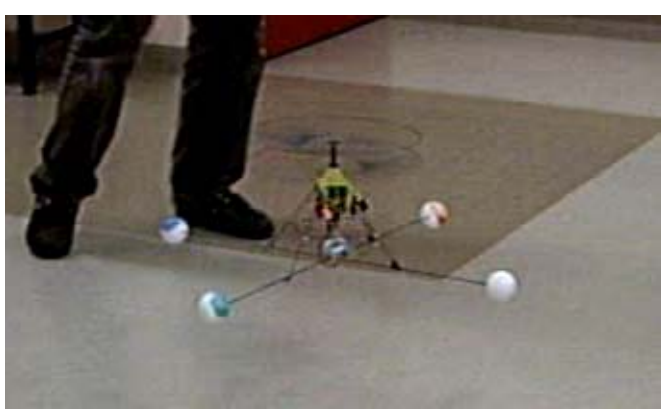

Fig. 12. CoaX in hover. A training frame was added for safety.

\section{B. Communication}

CoaX can be directly controlled by the user with a small IR remote control and/or with a PC graphical user interface via Bluetooth. Thanks to the passive stability of $\mathrm{CoaX}$, the simple manual control is sufficient to take-off. However, to increase the flight stability or to perform horizontal motion, the automatic embedded control algorithms can be switchedon. This decision is of course taken by the user through the 2 interface possibilities. In that way a hybrid manual-automatic control is possible. Typically the yaw control is left to the robot from the beginning, whereas the altitude control is turned-on as soon as the desired height is reached.

\section{Results Discussion}

CoaX is currently able to hover for short moments which validates the structure, the control electronics and the propulsion system. The lateral motion subsystem is effective, one can see from Fig. 10 the effect of the CoG shifting on the roll and pitch angles. The subsystem has about $10 \mathrm{~Hz}$ of bandwidth, but still suffer from mechanical hysteresis. This is clear if we look to the light blue curve (servo 2) and the dark blue one (roll) at $125 \mathrm{sec}$. We see no reaction on the latter until $127 \mathrm{sec}$ where the servo starts effectively moving the battery. Finally at $129 \mathrm{sec}$, the passive stability is overcome and the effect is clear on the roll angle. Figure 11 represents five successive flights, we were replacing by hand the helicopter between each flight in the center of the room. The first curve is the altitude controlled by a human. We were not risking high altitude flights as we have only one prototype. The second curve shows the yaw behavior under active control. It was effective and not problematic. In the third and forth curves, one can see that the propellers are providing some passive stability. The effect is less than what we expected, mainly because of flexibility saturation in the propellers. An enhanced version of the propeller is under development.

\section{CONCLUSion AND Future Work}

The focus of this paper is the development and integration of an indoor coaxial helicopter. A systematic design method was applied thanks to which we achieved up to $40 \%$ thrust margin and nearly $20 \mathrm{~min}$ autonomy while respecting the initial requirements. CoaX electronics are fully integrated with the necessary sensors, actuators and communication modules 

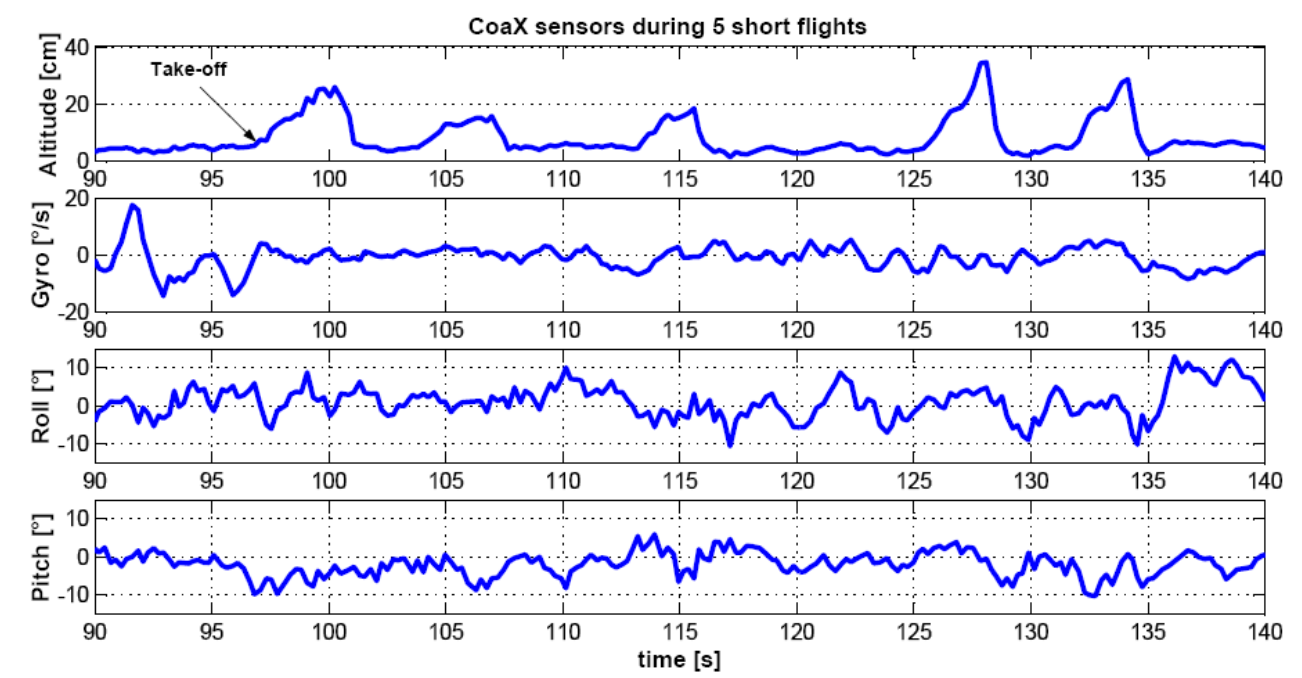

Fig. 11. Experiment: CoaX data in five successive short flights.

for autonomous operation. The structure is lightweight thanks to the use of special polyurethane at $0.7 \mathrm{Tn} / \mathrm{m}^{3}$ and carbon fiber. The propellers flexibility brings passive stability in orientation and the CoG shifting controls the lateral motion. CoaX is able to hover without any control for short moments. However, the propellers need mechanical and aerodynamical improvements. This will avoid the frequent breaks and the flexibility saturation. On the other hand, we will improve the CoG shifting mechanism to get less battery oscillations. In the future, an analytical model will be developed allowing the analysis of CoaX dynamics, especially the interaction between the flexible propellers and the CoG shifting subsystems. It will also allow the development of more advanced controllers. The fact that CoaX is passively stable, has a relatively simple mechanics and embedded control electronics together with a conformable thrust margin, shows that this is the way toward even smaller MFR with great potential.

\section{ACKNOWLEDGMENT}

The authors would like to thank André Noth for fruitful discussions about flying robots, Tarek Baaboura for the mechanical parts realization and Peter Bruehlmeier for PCB design. We would like to thank GCtronic for its contribution in electronics and mechatronics and finally all the students who worked or are working on the project.

\section{REFERENCES}

[1] G. Hoffmann et al., "The stanford testbed of autonomous rotorcraft for multi agent control (starmac)," in Proc. 23rd Digital Avionics Systems Conference (DASC'04), Salt Lake City, USA, Oct. 2004.

[2] I. Kroo et al., "The mesicopter: A miniature rotorcraft concept phase ii interim report," Stanford University, USA, 2000.

[3] X. Deng et al., "Attitude control for a micromechanical flying insect including thorax and sensor models," in Proc. International Conference on Robotics and Automation (ICRA'03), Teipei, Taiwan, 2003.

[4] F. Bohorquez, "Design, analysis and performance of a rotary wing mav," Alfred Gessow Rotorcraft Center, USA, 2001.

[5] The EPSON website. [Online]. Available: http://www.epson.co.jp/

[6] Aero-epfl. [Online]. Available: http://aero.epfl.ch/

[7] S. Bouabdallah and R. Siegwart, "Towards intelligent miniature flying robots," in Proc. of Field and Service Robotics, Port Douglas, Australia, 2005.

[8] Proxflyer. [Online]. Available: http://www.proxflyer.com/

[9] P. Samuel et al., "Design and testing of a rotary wing mav with an active structure for stability and control," in Proc. AHS Annual Forum 61, Grapevine, USA, 2005.

[10] Vti. [Online]. Available: http://www.vti.fi/productsen/productsen_2_1_3_ 1.html

[11] Ad. [Online]. Available: http://www.analog.com/en/prod/0,2877, ADXRS300,00.html

[12] Sharp. [Online]. Available: http://www.sharpsma.com/part.php?PartID= 3895 